\title{
PREVALENCE OF ANAPLASMA PHAGOCYTOPHILA AND BORRELIA BURGDORFERI IN IXODES PERSULCATUS TICKS FROM NORTHEASTERN CHINA
}

\author{
WU-CHUN CAO, QIU-MIN ZHAO, PAN-HE ZHANG, HONG YANG, XIAO-MING WU, BO-HAI WEN, XI-TAN ZHANG, \\ AND J. DIK F. HABBEMA \\ Deparment of Epidemiology, Beijing Institute of Microbiology and Epidemiology, Beijing, People's Republic of China; Center for \\ Decision Sciences in Tropical Disease Control, Department of Public Health, Erasmus University Rotterdam, The Netherlands
}

\begin{abstract}
A total of 1,345 Ixodes persulcatus ticks collected from northeastern China were investigated for the presence of Anaplasma phagocytophila and Borrelia burgdorferi by a nested polymerase chain reaction (PCR). Sixty-two (4.6\%) ticks were positive for A. phagocytophila and $454(33.8 \%)$ were positive for B. burgdorferi. Seven $(0.5 \%)$ were coinfected with both agents. Sequence analysis of 919-basepair PCR amplicons revealed three types of A. phagocytophila. Type 1 was identical to the published sequences of $A$. phagocytophilas responsible for human granulocytic ehrlichiosis (HGE). The other two variants differed from the HGE agent sequence at one and four positions, respectively. These findings imply that infection with $A$. phagocytophila poses a potential health threat to both humans and animals in northeastern China, and that ehrlichiosis should be considered in the differential diagnosis of febrile patients with a history of tick bite, particularly when clinical manifestations are atypical for Lyme disease.
\end{abstract}

\section{INTRODUCTION}

Human granulocytic ehrlichiosis (HGE) is an emerging tick-borne disease first recognized in the upper midwestern United States in $1994 .{ }^{1}$ Patients with HGE often present relatively nonspecific symptoms that include fever, myalgia, headache, chills, lethargy, arthralgia, leukopenia, thrombocytopenia, and a mild elevation in levels of transaminases. ${ }^{2,3}$ The etiologic agent of HGE is closely related to Ehrlichia phagocytophila and E. equi. ${ }^{4}$ However, these species are now considered one species, and they were renamed Anaplasma phagocytophila in a recent reclassification. ${ }^{5}$

Anaplasma phagocytophila is transmitted by genus Ixodes ticks, including I. scapularis, ${ }^{6,7}$ and I. pacificus ${ }^{8,9}$ in the United States, I. ricinus in Europe, ${ }^{10,11}$ and I. persulcatus in Asia. ${ }^{12}$ These ticks also serve as the vectors of Borrelia burgdorferi, the causative agent of Lyme disease. Coinfection of ticks with $A$. phagocytophila and B. burgdorferi has been increasingly reported in recent years. ${ }^{13-16}$ Northeastern China is highly endemic for Lyme disease, ${ }^{17}$ where the infection rate of $B$. burgdorferi in adult $I$. persulcatus ticks can be as high as $44.1 \%{ }^{18}$ Recently, the presence of $A$. phagocytophila in ticks from the region has been reported. ${ }^{12}$ Therefore, an extensive study was carried out to confirm the previous findings, and to investigate coinfection of $I$. persulcatus ticks with $A$. phagocytophila and B. Burgdorferi.

\section{MATERIALS AND METHODS}

Collection of ticks. Ixodes persulcatus ticks were collected by flagging vegetation from four areas in Inner Mongolia Autonomous Region and Heilongjiang Province in the summers of 1999 and 2001, respectively. All the collection sites are forested highlands belonging to Great Xing-An Mountains and Small Xing-An Mountains. Ticks were kept alive in a refrigerator until tested.

Extraction of DNA. After identification, ticks were soaked in $70 \%$ ethanol for a few minutes, and then rinsed three times in sterile water. Extraction of DNA was performed as previously described. ${ }^{19}$ Briefly, the ticks were placed into microtubes and mechanically disrupted with sterile scissors in $50 \mu \mathrm{L}$ of DNA extraction buffer (10 mM Tris, $\mathrm{pH}$ 8.0, 2 mM EDTA, $0.1 \%$ sodium dodecyl sulfate, $500 \mu \mathrm{g}$ of proteinase $\mathrm{K} / \mathrm{mL}$ ).
The samples were incubated for two hours at $56^{\circ} \mathrm{C}$ and then boiled at $100^{\circ} \mathrm{C}$ for 10 minutes to inactivate the proteinase $\mathrm{K}$. After centrifugation, the supernatant was transferred to fresh sterile microtubes and purified by extracting twice with an equal volume of phenol-chloroform before use.

Amplification by a polymerase chain reaction (PCR). A nested PCR was performed with primers designed to amplify the 16S rRNA gene of $A$. phagocytophila. ${ }^{12}$ Primers GE9f and GE10r, previously described by Chen and others, ${ }^{4}$ were used for the primary amplification. The PCR amplifications were performed in a volume of $30 \mu \mathrm{L}$ in a Perkin-Elmer (Norwalk, CT) model 2400 thermal cycler. An initial threeminute denaturation at $95^{\circ} \mathrm{C}$ was followed by 35 cycles at $94^{\circ} \mathrm{C}$ for 15 seconds, $55^{\circ} \mathrm{C}$ for 20 seconds, and $72^{\circ} \mathrm{C}$ for 15 seconds, and a final extension at $72^{\circ} \mathrm{C}$ for five minutes. In nested PCR, the components and conditions were similar to those for the primary amplification, except that primers GE9f and GE2 were used, ${ }^{20}$ and $1 \mu \mathrm{L}$ of the primary PCR product was used as the template. A positive control (a plasmid containing the 16S rRNA gene of the HGE agent (kindly provided by Dr. J. Stephen Dumler, Department of Pathology, The Johns Hopkins Medical Institutions, Baltimore, MD) and a negative control (distilled water) were included with each set of amplifications.

For amplification of B. burgdorferi DNA, a nested PCR was carried out with primers derived from the B. burgdorferi outer surface protein A (osp $A$ ) gene. ${ }^{21}$ For the primary amplification, $3 \mu \mathrm{L}$ of each template sample was amplified in a $30-\mu \mathrm{L}$ reaction mixture containing the primers $\mathrm{OA} 1$ and OA4. One microliter of the primary PCR product was then used as the template in a second $30-\mu \mathrm{L}$ reaction mixture with primers BSL and OA4. The PCR amplifications were performed in a Perkin-Elmer model 2400 thermal cycler using the following protocol: preheating at $95^{\circ} \mathrm{C}$ for three minutes, followed by 40 cycles at $94^{\circ} \mathrm{C}$ for 20 seconds, $50^{\circ} \mathrm{C}$ for 20 seconds, and $72^{\circ} \mathrm{C}$ for 30 seconds, and a final extension at $72^{\circ} \mathrm{C}$ for seven minutes. In parallel with each amplification of tick specimens, DNA from a B. burgdorferi isolate was used as a positive control and distilled water was used as a negative control.

Reaction products were separated by agarose gel electrophoresis, stained with ethidium bromide, and visualized under UV light. To minimize contamination, DNA extraction, the 
reagent setup, amplification, and agarose gel electrophoresis were performed in separate rooms.

Cloning of PCR products and DNA sequencing. The products of nested amplification were collected, and the purified DNA fragments were then cloned into the plasmid vector pGEM-T (Promega Corp., Madison, WI) and transformed into competent cells (Escherichia coli XL1-Blue) according to the manufacturer's instructions. The recombinant plasmids were extracted and purified from overnight cultures using QIA prep Spin Miniprep Kit (Qiagen, Valencia, CA). The nucleotide sequences of the plasmid inserts were determined by a dideoxynucleotide cycle sequencing method with an automated DNA sequencer (ABI PRISM 377; Perkin-Elmer).

\section{RESULTS}

A total of 1,345 I. persulcatus ticks were examined for the presence of $A$. phagocytophila and B. burgdorferi sensu lato. The distribution of ticks according to origin, sex and stage, and infection status is shown in Table 1. Anaplasma phagocytophila were detected in $4.6 \%$ of the ticks. Of 643 I. persulcatus ticks collected from the Wuerqihan and Moerdaoga forestry farms in the Great Xingan Mountains of Inner Mongolia, $40(6.2 \%)$ contained $A$. phagocytophila. The difference in infection rates among male, female, and nymphal ticks was not significant $\left(\chi^{2}=1.69\right.$, degrees of freedom $[\mathrm{df}]=2, P=$ $0.43)$. The frequency of positive ticks from the Weihe forestry farm in the Small Xingan Mountains of Heilongjiang was $3.1 \%$ (22 of 702 ticks), which was significantly lower than that at Inner Mongolia $\left(\chi^{2}=6.59, \mathrm{df}=1, P=0.01\right)$. Although $A$. phagocytophila DNA was not detected in nymphal ticks in this area, the frequency of positive ticks was not statistically different regardless of sex or stage $\left(\chi^{2}=3.71\right.$, df $=2, P=$ $0.16)$.

The prevalence of $B$. burgdorferi in ticks from the Great Xingan Mountains was 27.7\% (178 of 643 ticks). The infection rate in adults $(47.9 \%)$ was significantly higher than that in nymphs $(8.0 \%)\left(\chi^{2}=29.28, \mathrm{df}=1, P<0.001\right)$. In contrast to infection with $A$. phagocytophila, infection with $B$. burgdorferi was more prevalent in ticks in the Small Xingan Mountains $(39.3 \%)$ than that in the Great Xingan Mountains $\left(\chi^{2}=\right.$ 19.80 , df $=1, P<0.001)$. There was a significant difference in $B$. burgdorferi infection between adult and nymphal ticks from the Small Xingan Mountains. $\left(\chi^{2}=8.95\right.$, df $=1, P=$ 0.003). Among 62 A. phagocytophila-positive I. persulcatus ticks, seven were coinfected with $B$. burgdorferi. The overall coinfection rate was $0.5 \%$. No difference in proportions of coinfected ticks was demonstrated between the two studied areas.

Ten ticks positive for $A$. phagocytophila by the nested PCR were randomly selected for sequence analysis. Of the 10 positive specimens, six ( 3 males, 2 females, and 1 nymph) were from the Great Xingan Mountains and four (2 males and 2 females) were from the Small Xingan Mountains. A 919basepair nucleotide sequence amplified with primer pair GE9f and GE10r ${ }^{4}$ was obtained from each tick specimen. Sequence analysis of the 10 PCR amplicons revealed three variants of $A$. phagocytophila (Table 2 ). The nucleotide sequences from two male and one female ticks from the Great Xingan Mountains and one male tick from the Small Xingan Mountains were identical to the published sequences of the HGE agent. In addition, two sequence variants were detected. Variant 1, isolated from a male, a female, and a nymphal tick from the Great Xingan Mountains, had a $\mathrm{T}$ instead of an A at position 81 according to the corresponding sequence of the HGE agent (GenBank accession no. U02521). Variant 2, isolated from a male and two female ticks from the Small Xingan Mountains, differed from the HGE agent sequence by four bases at position $76,77,80$, and 84 (Table 2).

\section{DISCUSSION}

The prevalence of $A$. phagocytophila infection in I. persulcatus ticks was investigated in the forest areas of northeastern China where Lyme disease the tick-borne encephalitis are endemic. As a result, $4.6 \%$ ticks were found to be infected, which further confirmed the existence of A. phagocytophila in the region. However, the overall infection rate of ticks determined in the present study is remarkably higher than that in our previous investigation. ${ }^{12}$ A significant difference in the positive rate was also demonstrated between ticks from the Great Xingan Mountains and ticks from the Small Xingan Mountains. Recently, discrepant infection rates of $A$. phagocytophila in ticks from different areas around the world have been reported. The prevalence of $A$. phagocytophila infection was $0.8 \%$ in adult I. pacificus from California, ${ }^{7}$ and in freeliving adult $I$. ricinus from areas endemic for tick-borne fever in Switzerland, ${ }^{11}$ which are comparable to our previous findings. ${ }^{12}$ Higher prevalences were reported in I. scapularis and I. pacificus in the United States, ${ }^{5,6,8,9,22}$ and in I. ricinus in Europe. ${ }^{10,11,15,16,23}$ This discrepancy in positive rates could be attributable to differences in sampling approaches, tick species, geographic and seasonal variations of infected ticks, or to

TABLE 1

Results of the nested polymerase chain reaction for the identification of Anaplasma phagocytophila and Borrelia burgdorferi in Ixodes persulcatus ticks from northeastern China

\begin{tabular}{llcccc}
\hline \multirow{2}{*}{ Origin } & & & \multicolumn{2}{c}{ No. (\%) of ticks infected with } \\
\cline { 3 - 6 } Great Xingan Mountains & Sex or stage & No. of ticks & A. phagocytophila & B. burgdorferi & A. phagocytophila and B. burgdorferi \\
& Male & 323 & $18(5.6)$ & $106(32.8)$ & $3(0.9)$ \\
Small Xingan Mountains & Female & 199 & $16(8.0)$ & $63(31.7)$ & 0 \\
& Nymph & 121 & $6(5.0)$ & $9(7.4)$ & $1(0.8)$ \\
& Male & 283 & $12(4.2)$ & $108(38.2)$ & $1(0.4)$ \\
Total & Female & 341 & $10(2.9)$ & $150(44.0)$ & $2(0.6)$ \\
& Nymph & 78 & 0 & $18(23.1)$ & 0 \\
\hline
\end{tabular}


TABLE 2

Comparison of partial 16S rRNA gene sequences of Anaplasma phagocytophila in Ixodes persulcatus from northeastern China with published sequences of $A$. phagocytophila of different origins

\begin{tabular}{|c|c|c|c|c|c|c|c|c|c|}
\hline \multirow[b]{2}{*}{ Geographic origin } & \multirow[b]{2}{*}{ Biological origin } & \multicolumn{6}{|c|}{ Nucleotide difference at position* } & \multirow[b]{2}{*}{ GenBank accession no. } & \multirow[b]{2}{*}{ Reference } \\
\hline & & 76 & 77 & 80 & 81 & 84 & 886 & & \\
\hline USA & Human & A & A & A & A & $\mathrm{G}$ & $\mathrm{G}$ & U02521 & 4 \\
\hline USA & Horse & A & A & A & A & A & $-\dagger$ & M73223 & 31 \\
\hline USA & Goat & A & A & A & A & A & $-\dagger$ & M73220 & 31 \\
\hline USA & Roe deer & G & A & A & A & A & G & AF384213 & 20 \\
\hline China & I. persulcatus & A & A & A & $\mathrm{T}$ & $\mathrm{G}$ & G & AY079425 & This study \\
\hline China & I. persulcatus & G & G & G & A & A & G & AF205140 & This study \\
\hline Sweden & I. ricinus & A & A & A & A & $\mathrm{G}$ & G & AJ242785 & 23 \\
\hline Sweden & I. ricinus & G & A & A & A & G & G & AJ242783 & 23 \\
\hline Sweden & I. ricinus & G & A & A & A & A & G & AJ242784 & 23 \\
\hline Canada & I. scapularis & $\mathrm{G}$ & A & A & A & A & $\mathrm{G}$ & AF311343 & 30 \\
\hline
\end{tabular}

* The position of the nucleotide relative to the sequence of the agent for human granulocytic ehrlichiosis (HGE) reported by Chen and others.

$\dagger-$ indicates no nucleotide corresponds to the HGE agent; a gap was required at this position to align the adjacent sequence.

limits of PCR sensitivity. A study carried out in southern Norway showed that tick samples taken from different locations and at different time points might have different rates of infection with $A$. phagocytophila. ${ }^{24}$ All these findings imply that estimates based on spot investigations may have only local and temporary applicability, and have limited value in forming public health policy.

Ixodes persulcatus ticks are distributed over an extensive area from Russia to eastern Asia, where approximately onefifth of the human population of the world resides. The results obtained in the present survey demonstrate that $A$. phagocytophila infection poses a potential health threat to both humans and animals where $I$. persulcatus is abundant, and should be useful in alerting public health officials and clinicians about the presence of ehrlichiosis in northeastern China. In contrast to our study, an investigation conducted in the Baltic region of Russia failed to demonstrate A. phagocytophila infection in I. persulcatus. ${ }^{25}$ In southern Germany, ehrlichia-positive ticks were only found in one of five surveyed regions, arguing that the distribution of $A$. phagocytophila seems to be focal. ${ }^{26}$ Further epidemiologic studies are required to clarify the diversity of $A$. phagocytophila, to identify its natural foci, and especially to define potential human and animal infection risks following tick bites in areas infested with I. persulcatus.

In forest areas of northeastern China, I. persulcatus is the most abundant tick species and is responsible for the majority of tick bites in humans. This tick species infests multiple animal hosts, and therefore, may acquire more than one pathogen from different reservoirs. Coinfection of ixodid ticks with $A$. phagocytophila and B. burgdorferi has been reported in the United States and many European countries with various prevalences.9,10,15,16,24 In our study, seven of 62 ehrlichiapositive ticks were found to harbor both $A$. phagocytophila and B. burgdorferi. Coexistence of the two pathogens in $I$. persulcatus ticks from Asia has not been previously reported. This finding suggests that humans may become coinfected with the two pathogens as a consequence of a single tick bite. In fact, simultaneous human infection with the two agents has already been reported, ${ }^{27}$ and it may lead to variations in clinical symptoms and signs. ${ }^{28,29}$

If one considers that A. phagocytophila can cause immunosuppression in its mammalian hosts, coinfection with two or more tick-borne agents may aggravate the clinical pictures of Lyme disease and tick-borne encephalitis. The identification of A. phagocytophila in I. persulcatus, and the finding of coinfection with $B$. burgdorferi in the current study imply that the possible occurrence of ehrlichiosis should be considered in the differential diagnosis of febrile patients with a history of tick bite in the forest areas of northeastern China, particularly when clinical manifestations are atypical for Lyme disease. Unfortunately, the B. burgdorferi genospecies was not determined in the present investigation. A previous study reported $64.4 \%$ B. garinii and $35.6 \%$ B. afzelii based on a PCRrestriction fragment length polymorphism analysis of $45 B$. burgdorferi isolates from this area. ${ }^{18}$ Whether the coinfection was associated with $B$. burgdorferi genospecies remains to be determined.

The nucleotide sequences of PCR products from tick samples were all identified as part of $A$. phagocytophila $16 \mathrm{~S}$ rRNA gene, and showed high level of identity (99.6-100\%) with published sequences of the HGE agent (Table 2). Three types of $A$. phagocytophila sequences were found: one with the identical sequence of the HGE agent, and two variants with nucleotide differences from the HGE agent by one and four basepairs, respectively. A variable region was found near the $5^{\prime}$ end of $16 \mathrm{~S}$ rRNA gene at the position from 76 to 84 (according to HGE agent [GenBank accession no. U02521]). This result is consistent with findings of previous studies in other places, in which nucleotide differences were also identified in this variable region but at different positions. ${ }^{13,20,23,30,31}$ It remains to be determined whether each molecular variant of $A$. phagocytophila can cause a disease in humans or animals.

Received June 20, 2002. Accepted for publication January 3, 2003.

Acknowledgments: We are grateful to Dr. Rong-man Xu for identifying ticks and reading the manuscript, and to Dr. Jian-hua Zhu and Dr. Shan-hu Chen (The Central Anti-Epidemic Station of the Great Xingan Mountains) for assistance in collection of ticks.

Financial support: This study was supported by the National Natural Science Foundation of China (grant no. 39970655) and the Beijing Natural Science Foundation (grant no. 7992029).

Authors' addresses: Wu-Chun Cao, Qiu-Min Zhao, Pan-He Zhang, Hong Yang, Xiao-Ming Wu, Bo-Hai Wen, and Xi-Tan Zhang, Beijing Institute of Microbiology and Epidemiology, 20 Dong-Da-Jie Street, Fengtai District, Beijing 100071, People's Republic of China. J. Dik F. Habbema, Center for Decision Sciences in Tropical Disease Control, Department of Public Health, Faculty of Medicine and Health Sci- 
ences, Erasmus University Rotterdam, PO Box 1738, 3000 DR Rotterdam, The Netherlands.

Reprint requests: Dr. Wu-Chun Cao, Beijing Institute of Microbiology and Epidemiology, 20 Dong-Da-Jie Street, Fengtai District, Beijing 100071, People's Republic of China, Telephone/Fax: 86-1063812060, E-mail: caowc@nic.bmi.ac.cn

\section{REFERENCES}

1. Bakken JS, Dumler JS, Chen SM, Eckman MR, Van Etta LL, Walker DH, 1994. Human granulocytic ehrlichiosis in the upper midwest United States. A new species emerging? JAMA 272: 212-218.

2. Bakken JS, Krueth J, Wilson-Nordskog C, Tilden RL, Asanovich K, Dumler JS, 1996. Clinical and laboratory characteristics of human granulocytic ehrlichiosis. JAMA 275: 199-205.

3. Walker DH, Dumler JS, 1996. Emergence of the ehrlichioses as human health problems. Emerg Infect Dis 2: 18-29.

4. Chen SM, Dumler JS, Bakken JS, Walker DH, 1994. Identification of a granulocytotropic Ehrlichia species as the etiologic agent of human disease. J Clin Microbiol 32: 589-595.

5. Dumler JS, Barbet AF, Bekker CP, Dasch GA, Palmer GH, Ray SC, Rikihisa Y, Rurangirwa FR, 2001. Reorganization of genera in the families Rickettsiaceae and Anaplasmataceae in the order Rickettsiales: unification of some species of Ehrlichia with Anaplasma, Cowdria with Ehrlichia and Ehrlichia with Neorickettsia, descriptions of six new species combinations and designation of Ehrlichia equi and 'HGE agent' as subjective synonyms of Ehrlichia phagocytophila. Int J Syst Evol Microbiol 51: 2145-2165.

6. Magnarelli LA, Stafford KC III, Mather TN, Yeh MT, Horn KD, Dumler JS, 1995. Hemocytic rickettsia-like organisms in ticks: serologic reactivity with antisera to ehrlichiae and detection of DNA of agent of human granulocytic ehrlichiosis by PCR. $J$ Clin Microbiol 33: 2710-2714.

7. Pancholi P, Kolbert CP, Mitchell PD, Reed KD, Dumler JS, Bakken JS, Telford SR, Persing DH, 1995. Ixodes dammini as a potential vector of human granulocytic ehrlichiosis. J Infect Dis 72: 1007-1012.

8. Barlough JE, Madigan JE, Kramer VL, Clover JR, Hui LT, Webb JP, Vredevoe LK, 1997. Ehrlichia phagocytophila genogroup rickettsiae in ixodid ticks from California collected in 1995 and 1996. J Clin Microbiol 35: 2018-2021.

9. Kramer VL, Randolph MP, Hui LT, Irwin WE, Gutierrez AG, Vugia DJ, 1999. Detection of the agents of human ehrlichioses in ixodid ticks from California. Am J Trop Med Hyg 60: 62-65.

10. Gug E, Tasker S, Joynson DHM, 1998. Detection of the agent of human granulocytic ehrlichiosis (HGE) in UK ticks using polymerase chain reaction. Epidemiol Infect 21: 681-683.

11. Pusterla N, Leutenegger CM, Huder JB, Weber R, Braun U, Lutz $H, 1999$. Evidence of the human granulocytic ehrlichiosis agent in Ixodes ricinus ticks in Switzerland. J Clin Microbiol 37: 1332-1334.

12. Cao W-C, Zhao Q-M, Zhang P-H, Dumler JS, Zhang X-T, Fang L-Q, Yang H, 2000. Granulocytic ehrlichiae in Ixodes persulcatus ticks from an area in China where Lyme disease is endemic. J Clin Microbiol 38: 4208-4210.

13. Baumgarten BU, Röllinghoff M, Bogdan C, 1999. Prevalence of Borrelia burgdorferi and granulocytic and monocytic ehrlichiae in Ixodes ricinus ticks from southern Germany. J Clin Microbiol 37: 3448-3451.

14. Leutenegger CM, Pusterla N, Mislin CN, Weber R, Lutz H, 1999. Molecular evidence of coinfection of ticks with Borrelia burgdorferi sensu lato and the human granulocytic ehrlichiosis agent in Switzerland. J Clin Microbiol 37: 3390-3391.
15. Schouls LM, van de Pol I, Rijpkema SGT, Schot CS, 1999. Detection and identification of Ehrlichia, Borrelia burgdorferi sensu lato, and Bartonella species in Dutch Ixodes ricinus ticks. J Clin Microbiol 37: 2215-2222.

16. Christova I, Schouls L, Van de Pol I, Park J, Panayotov S, Lefterova V, Kantardjiev T, Dumler DS, 2001. High prevalence of granulocytic ehrlichiae and Borrelia burgdorferi snesu lato in Ixodes ricinus ticks from Bulgaria. J Clin Microbiol 39: 4172-4174.

17. Ai CX, Zhang WF, Zhao JH, 1994. Sero-epidemiology of Lyme disease in an endemic area in China. Microbiol Immunol 38: 505-509.

18. Takada N, Fubito I, Fajita H, Wang H-P, Wang J-C, Masuzawa T, 1998. Lyme disease spirochetes in ticks from northeastern China. J Parasitol 84: 499-504.

19. Cao W-C, Gao Y-M, Zhang P-H, Zhang X-T, Dai Q-H, Dumler JS, Fang L-Q, Yang H, 2000. Identification of Ehrlichia chaffeensis by nested PCR in ticks from southern China. J Clin Microbiol 38: 2778-2780.

20. Massung RF, Slater K, Owens JH, Nicholson WL, Mather TN, Solberg VB, Olson JG, 1998. Nested PCR assay for detection of granulocytic ehrlichiae. J Clin Microbiol 36: 1090-1095.

21. Vasiliu V, Herzer P, Rossler D, Lehnert G, Wilske B, 1998. Heterogeneity of Borrelia burgdorferi sensu lato demonstrated by an Osp-A-type-specific PCR in synovial fluid from patients with Lyme arthritis. Med Microbiol Immunol (Berl) 187: 97102.

22. Barlough JE, Madigan JE, Turoff DR, Clover JR, Shelly SM, Dumler JS, 1997. An Ehrlichia strain from a llama (Lama glama) and llama-associated ticks (Ixodes pacificus). J Clin Microbiol 35: 1005-1007.

23. Von Stedingk LV, Gürtelschmid M, Hanson HS, Gustafson R, Dotevall L, Olsson Engvall E, Granström M, 1997. The human granulocytic ehrlichiosis (HGE) agent in Swedish ticks. Clin Microbiol Infect 3: 573-574.

24. Jenkins A, Kristiansen B-E, Allum A-G, Aakre RK, Srand L, Kleveland EJ, Van De Pol I, Schouls L, 2001. Borrelia burgdorferi sensu lato and ehrlichia spp. in Ixodes ticks from southern Norway. J Clin Microbiol 39: 3666-3671.

25. Alekseev AN, Dubinina HV, van de Pol, I, Schouls LM, 2001. Identification of Ehrlichia spp. and Borrelia burgdorferi in Ixodes ticks in the Baltic regions of Russia. J Clin Microbiol 39: 2237-2242.

26. Fingerle V, Munderloh UG, Liegl G, Wilske B, 1999. Coexistence of ehrlichiae of the phagocytophila group with Borrelia burgdorferi in Ixodes ricinus from southern Germany. Med Microbiol Immunol 188: 145-149.

27. Duffy J, Pittlekow MR, Kolbert CP, Rutledge BJ, Persing DH, 1997. Coinfection with Borrelia burgdorferi and the agent of human granulocytic ehrlichiosis (letter). Lancet 349: 399.

28. Nadelman RB, Horowitz HW, Hsieh T-C, Wu JM, AgueroRosenfeld ME, Schwartz I, Nowakowski J, Varde S, Wormser GP, 1997. Simultaneous human granulocytic ehrlichiosis and Lyme borreliosis. $N$ Engl J Med 337: 27-30.

29. Weber R, Pusterla N, Loy M, Lutz H, 1998. Fever, leukopenia, and thrombocytopenia in a patient with acute Lyme borreliosis were due to human granulocytic ehrlichiosis. Clin Infect Dis 26: 253-254.

30. Drebot MA, Lindsay R, Barker IK, Artsob H, 2001. Characterization of a human granulocytic ehrlichiosis-like agent from Ixodes scapularis, Ontario, Canada. Emerg Infect Dis 7: 479480.

31. Anderson BE, Dawson J E, Jones DC, Wilson KH, 1991. Ehrlichia chaffeensis, a new species associated with human ehrlichiosis. J Clin Microbiol 29: 2838-2842 